\begin{tabular}{|c|c|}
\hline Title & $\begin{array}{l}\text { Quasi-automatic phase-control technique for chirp compensation of pulses with over-one-octave bandwidth-generation } \\
\text { of few- to mono-cycle optical pulses }\end{array}$ \\
\hline Author(s) & Y amashita, Mikio; Y amane, Keisaku; Morita, Ryuji \\
\hline Citation & $\begin{array}{l}\text { IEEE Journal of Selected Topics in Quantum Electronics, 12(2), 213-222 } \\
\text { https://doi.org/10.1109/SST QE.2006.871961 }\end{array}$ \\
\hline Issue Date & $2006-03$ \\
\hline Doc URL & http:/hdl.handle.net/2115/14392 \\
\hline Rights & $\begin{array}{l}\text { @2006 IEEE. Personal use of this material is permitted. However, permission to reprint/republish this material for } \\
\text { advertising or promotional purposes or for creating new collective works for resale or redistribution to servers or lists, } \\
\text { or to reuse any copyrighted component of this work in other works must be obtained from the IEEE, "IEEE Journal of } \\
\text { Selected Topics in Quantum Electronics, V olume 12, Issue 2, 2006, Page(s): } 213-222 "\end{array}$ \\
\hline Type & article \\
\hline File Information & 01632167.pdf \\
\hline
\end{tabular}

Instructions for use 


\title{
Quasi-Automatic Phase-Control Technique for Chirp Compensation of Pulses With Over-One-Octave Bandwidth-Generation of Few- to Mono-Cycle Optical Pulses
}

\author{
Mikio Yamashita, Keisaku Yamane, and Ryuji Morita
}

\author{
(Invited Paper)
}

\begin{abstract}
This paper introduces our self-recognition type of the computer-controlled spectral phase compensator (SRCSC), which consists of a greatly accurate phase manipulator with a spatial light modulator (SLM), a highly sensitive phase characterizer using a modified spectral phase interferometry for direct electric field reconstruction (M-SPIDER), and a computer for phase analysis and SLM control operating in the immediate feedback (FB) mode. The application of the SRCSC to adaptive compensation of various kinds of complicated spectral phases such as nonlinear chirped pulses with a weak intensity, induced-phase modulated pulses, photonic-crystal-fiber (PCF) output pulses, and nonlinear chirped pulses exceeding a 500-rad phase variation over-one-octave bandwidth demonstrated that the SRCSC is significantly useful for compensation of arbitrary nonlinear chirp and hence enables us to generate quasi-monocycle transform-limited (TL) pulses with a 2.8-fs duration. To the best of our knowledge, this 1.5-cycle pulse is the shortest single pulse with a clean temporal profile in the visible to near-infrared region.
\end{abstract}

Index Terms-Complicated nonlinear chirp compensation, optical pulse compression, over-one-octave bandwidth, quasiautomatic spectral phase control, quasi-monocycle pulse generation.

\section{INTRODUCTION}

W HEN one needs extremely short optical pulses such as few- to mono-cycle pulses, an optical pulse compression technique is usually employed. In such short-pulse compression, ultrabroadband pulses after being phase-modulated strongly exhibit often complicated nonlinear chirp, unlike ordinary linear chirp. Such nonlinear chirp is not compensated for by conventional passive dispersion elements (for example, a grating pair, a prism pair, chirped mirrors, and their combinations) because of interrelation between second-order and higher order phase dispersions of their compensators and their bandwidth limitations. In addition, few- to mono-cycle pulses generated as a result of pulse compression cannot be characterized correctly by a conventional technique of fringe resolved autocorrelation (FRAC) owing to the so called filter effect in second-harmonic generation (SHG) processes [1].

Manuscript received November 22, 2005; revised Jaunary 28, 2006.

M. Yamashita and R. Morita are with the Department of Applield Physics, Hokkaido University, Sapporo 060-8628, Japan (e-mail: mikio@eng.hokudai. ac.jp).

K. Yamane is with the Kawasaki Semiconductor Technology World Corporation, Fukui 918-8135, Japan.

Digital Object Identifier 10.1109/JSTQE.2006.871961
In recent years, high-powered pulse compression to few cycles was reported using a gas-filled hollow fiber, chirped mirrors, and an SHG frequency-resolved optical gating (SHGFROG) measurement [2]. Other groups reported cascaded pulse compression using two hollow fibers, chirped mirrors, a spatial light modulator (SLM) technique (see Section II), and a conventional spectral phase interferometry for direct electric field reconstruction (SPIDER) [3] measurement [4], [5]. These compressed results, however, were limited to pulses with a bandwidth narrower than one octave, and hence with a duration longer than two-optical cycles because of the bandwidth limitation of SHG-FROG and chirped mirrors and the low sensitivity of the conventional SPIDER. On the other hand, we reported pulse compression to the time region of the monocycle having an over-one-octave bandwidth using a single hollow fiber and a self-recognition type of the computer-controlled spectral-phase compensator (SRCSC; see Section II), where a combined system of the SLM technique, the modified SPIDER (M-SPIDER) [6], and the computer analysis and feedback (FB) technique was employed [7], [8]. The SLM technique, which is usually employed for pulse shaping [9], has advantages of the over-one-octave bandwidth, the programmable compensation for arbitrary spectral phase, and the quasi-real-time operation, while it has a disadvantage of the low throughput compared with that of chirped mirrors. And, it seems that the maximum bandwidth, the energy limitation, and the maximum group-delay compensation of the present SLM technique are 390-1200 nm, several $10 \mu \mathrm{J} /$ pulse, and several picoseconds, respectively. The acoustooptic modulator (AOM) technique [10] and the flexible mirror technique [11], both, are also employed for programmable phase compensation. However, the former cannot be applied for the compression of pulses shorter than 10 fs due to high dispersion of a long AOM crystal [10]. The latter has low spatial and phase resolutions, cumbersome and imperfect phase calibration of membrane deflection, and high deflection losses [11]. The first closed-loop FB control of the spectral phase by the SLM technique was done by monitoring the SHG intensity [12]. However, the time required to run the chirp compensation experiment for the generation of 11 -fs pulses after about 1000 iterations was roughly $15 \mathrm{~min}$. In addition, for shorter pulse compression in the near-octave bandwidth and few-cycle region, the SHG intensity cannot be the unique target and cannot always define a minimized pulse [1]. 


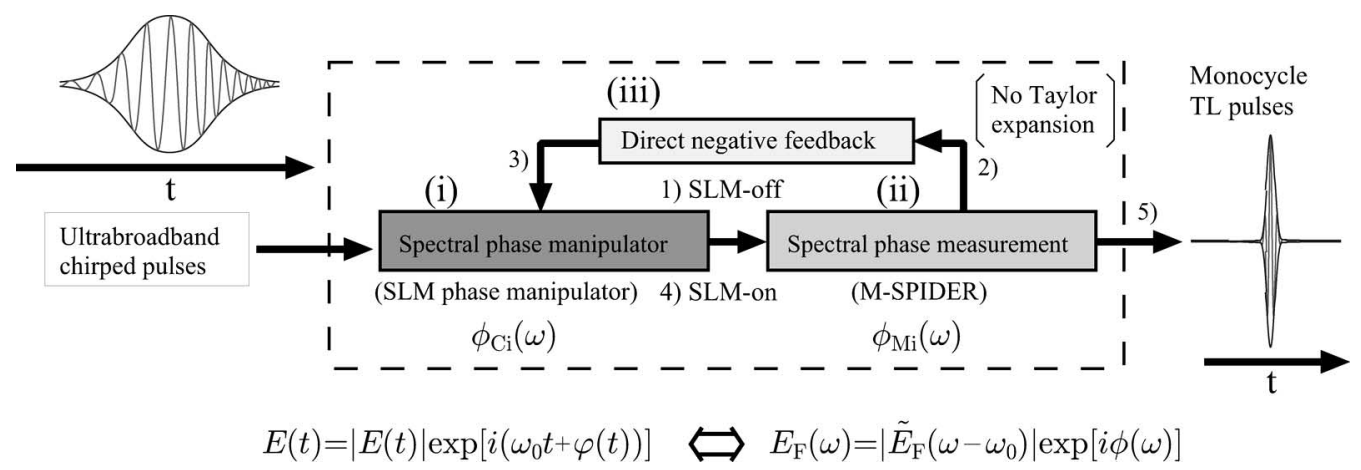

Fig. 1. Basic concept for SRCSC. SLM, spatial light modulator; M-SPIDER, modified spectral phase interferometry for direct electric field reconstruction. First, the spectral phase $\phi_{\mathrm{M} 1}(\omega)$ of ultrabroadband pulses passing through a phase manipulator with SLM-off is measured by M-SPIDER. Then, the first measured spectral phase is fed back negatively to the manipulator $\left(\phi_{\mathrm{C} 1}(\omega)=-\phi_{\mathrm{M} 1}(\omega)\right)$ and the output spectral phase $\phi_{\mathrm{M} 2}(\omega)$ is measured. The closed loop is repeated $\left(\phi_{\mathrm{C} 2}(\omega)=-\phi_{\mathrm{M} 1}(\omega)-\phi_{\mathrm{M} 2}(\omega)\right)$. Finally, the measured output spectral phase $\phi_{\mathrm{M} 3}(\omega)$ indicates TL pulses $\left(\phi_{\mathrm{M} 3}(\omega)=\phi_{\mathrm{TL}}(\omega)\right)$.

The purpose of this paper is to introduce our recently developed SRCSC [7], [8], [13]-[17]; that is, the paper shows that its quasi-automatic active compensator enables us to compensate for various kinds of nonlinear chirps of pulses with near- or over-one-octave bandwidths from different types of fibers. As a result, shortest compressed pulses are reported to be in the monocycle region, which will be useful for a direct study on the interaction dynamics between the matter and the temporal electric field wave packet (not the temporal intensity pulse), including the visualization and control of the electron motion [18].

\section{BASIC CONCEPT AND PRACTICAL SYSTEM: COMBINATION of Phase MANipulation, PHASE CHARACTERIZATION, AND COMPUTER ANALYSIS AND CONTROL}

Fig. 1 shows a schematic of basic concept of the selfrecognition type of the computer-controlled spectral-phase compensator (SRCSC). The "self-recognition type" implies that the SRCSC has two main functions of not only the computer control phase compensation but also the quasi-automatic measurement and FB of the unknown spectral phase. The function of the quasi-automatic compensation for the unknown spectral phase is significantly useful for users who are not familiar with optics. The SRCSC consists of a combination of a greatly accurate phase manipulator with an SLM [19]-[21], a highly sensitive phase characterizer using an M-SPIDER [6], [13], and a computer for phase analysis and SLM driving. The principle of the SRCSC is based on the precise measurement and manipulation of the spectral phase $\phi(\omega)$ of nonlinear chirped pulses in the frequency $\omega(x)$ region projected to a spatial coordinate $x$, utilizing the temporal (time $t$ )-spatial [frequency $\omega(x)$ ] Fourier transform (FT) by a spectroscopic dispersion system. In principle, arbitrary nonlinear chirp is compensated for by only the one immediate FB of the negative of the measured $\phi(\omega)$ over the entire spectral range without the need of the cumbersome Taylor expansion with different orders of phase dispersions, and hence the transform-limited (TL) pulse is accomplished.

Since the first experimental demonstration of the SRCSC was carried out for self-phase modulated pulses with a weak intensity from a fused-silica fiber in 2002 [13], the SRCSC has been applied for pulses with different nonlinear chirps [7], [8], [14]-[17]. Fig. 2 shows a practical system of the improved

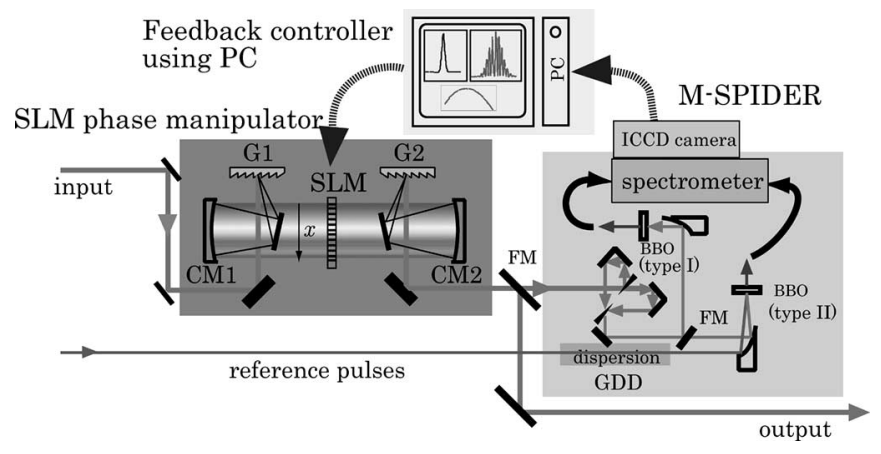

Fig. 2. Practical system of the SRCSC. Nonlinearly chirped pulses pass through the phase manipulator with gratings $(\mathrm{G} 1, \mathrm{G} 2)$, concave mirrors (CM1, CM2), and an SLM. Output spectral phase under SLM-off is measured by MSPIDER with intense cross-reference pulses, which are chirped by a dispersive glass (GDD). M-SPIDER consists of a Michelson interferometer to make a delay time $\tau_{\mathrm{d}}$, thin nonlinear crystals (BBOs), and an ICCD camera attached spectrometer. Feedback controller using a PC analyzes SPIDER signals to get the spectral phase and feeds its negative phase to SLM. FM, flipped mirror.

SRCSC, which was employed for the first generation of quasimonocycle pulses with a 3.3-fs duration in 2003 [7]. The phase manipulator with the so-called 4- $f$ configuration [9], [21] consists of a specifically designed liquid-crystal SLM (with a pixel number of 648, width of $97 \mu \mathrm{m}$, a gray-scale resolution of 192 , a transmission of $80 \%$ at $800 \mathrm{~nm}$ ), two aluminum-coated gratings (G1 and G2: a blaze wavelength of $500 \mathrm{~nm}$, a groove density of 150 lines $/ \mathrm{mm}$ ), and two aluminum-coated plane and concave (CM1 and CM2: focal length of $350 \mathrm{~mm}$ ) mirrors. The typical throughput is about $30 \%$. The input temporal chirped pulse is spatially Fourier transformed along the $x$-axis (see Fig. 2) at the SLM plane by G1 and CM1. Then, each phase $\phi\left(\omega_{i}\right)$ of different frequency components $\omega_{i}$ of the transformed electric field $E(\omega)$ is shifted independently by passing through the liquid crystal in each pixel, which was applied by a dc electric field so as to compensate for the chirp. Subsequently, the phase modulated pulse is inversely Fourier transformed by CM2 and G2 to get the temporal TL pulse. The influence of the second-order diffraction from gratings was avoided by suitable selection of the blaze wavelength [7]. The use of a prism pair [19] instead of a grating pair has advantages of no influence of the secondorder diffraction and the better throughput, and a disadvantage of large higher-order dispersions due to prism glasses. 
For the quasi-real-time measurement of the spectral phase $\phi(\omega)$, the output pulse from the phase manipulator is injected into the M-SPIDER, where two replicas with delay time $\tau_{\mathrm{d}}$ are produced by a Michelson interferometer. The replicas are upconverted with the external reference chirped pulse in a $20-\mu \mathrm{m}$ thick $\beta$-barium borate crystal (BBO type II, $45^{\circ}$ ). The interferogram [the SPIDER signal $D(\omega)$ ] with a spectral shear $\Omega / 2 \pi$ is measured by a highly wavelength-resolved spectrometer (1200 lines/mm; resolution less than $0.05 \mathrm{~nm}$ at $400 \mathrm{~nm}$ ) with an intensified charge-coupled device (ICCD). The availability of the external intensified reference pulse in the M-SPIDER, unlike the conventional SPIDER, provides the dramatically higher sensitivity [6]. The synchronously gated ICCD is cooled to $-25^{\circ} \mathrm{C}$ to increase the signal-to-noise ratio. The addition of the $\mathrm{SH}$ arm for independent measurement of the SH interferogram permits highly reproducible measurements.

The inverse FT of the SPIDER signal $D(\omega)$, which is the interference between two sum-frequency waves with slightly different frequency by $\Omega / 2 \pi$, enables us to have the so-called + ac component $D_{+ \text {ac }}(\tau)$ containing the phase difference term of $\theta^{\prime}(\omega)=\phi(\omega)-\phi(\omega-\Omega)+\tau_{\mathrm{d}} \omega$ [3]. After the subtraction of the $\tau_{\mathrm{d}} \omega$ term, the integral of the $\theta(\omega) / \Omega \cong[\phi(\omega)-\phi(\omega-$ $\Omega)] / \Omega$ with respect to $\omega$ gives the required spectral phase $\phi(\omega)$. This analytical process is performed directly by a computer. The negative phase $-\phi(\omega)$ is then programmably fed back to the SLM driver by the computer.

Accordingly, first, the spectral phase $\phi_{\mathrm{M} 1}(\omega)$ of input chirped pulses passing through the phase manipulator with the SLMswitch off is measured by the M-SPIDER. Then, the first measured spectral phase is fed back negatively to the manipulator $\left(\phi_{\mathrm{C} 1}(\omega)=-\phi_{\mathrm{M} 1}(\omega)\right)$ and the output spectral phase $\phi_{\mathrm{M} 2}(\omega)$ is measured. The closed loop is then repeated $\left(\phi_{\mathrm{C} 2}(\omega)=\right.$ $\left.-\phi_{\mathrm{M} 1}(\omega)-\phi_{\mathrm{M} 2}(\omega)\right)$. Finally, the measured output spectral phase $\phi_{\mathrm{M} 3}(\omega)$ indicates TL pulses $\left(\phi_{\mathrm{M} 3}(\omega)=\phi_{\mathrm{TL}}(\omega)\right)$. Typically, it takes few minutes for one closed loop because of the need of several automatic rotations of the grating in the spectrometer for ultrabroadband pulses.

The sophisticated SRCSC operates quasi-automatically but not completely. This is due to the fact that in the FT analysis of the SPIDER signal, the manual decision of the minimum $\tau_{\min }$ and maximum $\tau_{\max }$ values for the squared filter function is always needed for ultrabroadband pulses with fine spectral structures when the +ac component of $D_{+\mathrm{ac}}(\tau)$ is extracted. To overcome this problem, recently it has been demonstrated that the application of the wavelet transform (WT) method for the SPIDER signal analysis [22] instead of the conventional FT method enables us to operate in the complete automatic mode [23].

\section{APPLICATION FOR DifFERENT NONLINEARLY CHIRPED PULSES}

\section{A. Conventional Glass Fiber Experiments: Self-Phase Modulation (SPM) and Induced-Phase Modulation (IPM)}

1) SPM: The experiment of the first demonstrative SRCSC was carried out for self-phase-modulated pulses with low energy of $8.2 \mathrm{~nJ} /$ pulse at a $1-\mathrm{kHz}$ repetition rate (this pulse measure-
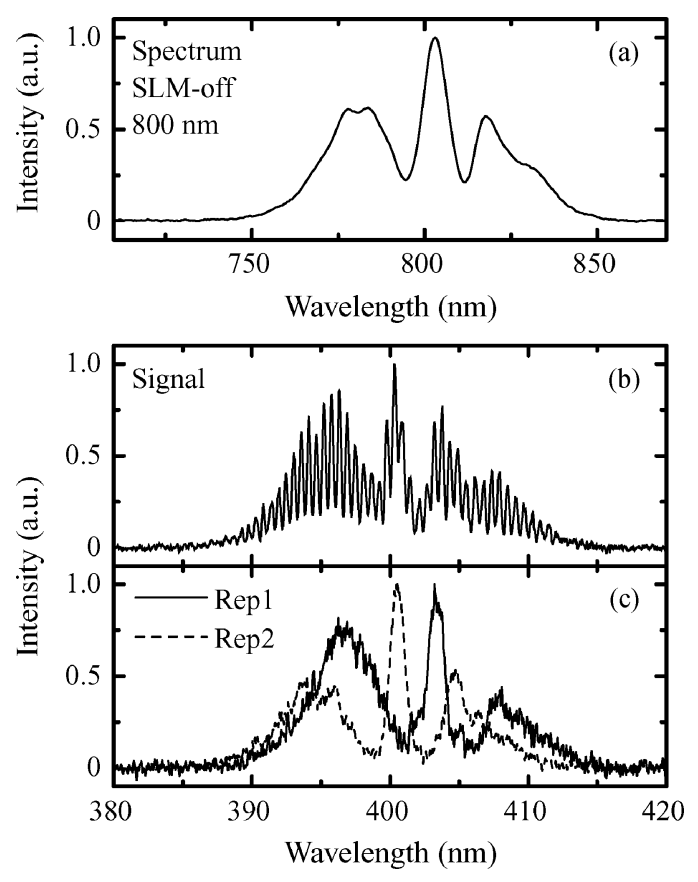

Fig. 3. SPM experiment. SLM-off case. (a) 800-nm glass-fiber output spectrum; (b) SPIDER signal; (c) replica spectra.

ment sensitivity corresponds to that of the conventional FRAC method), which were the output from a 3-mm-long fused-silica fiber [13]. Fiber input pulses of a 80-fs duration and 41-nJ energy at $800 \mathrm{~nm}$ were broadened from 730 to $870 \mathrm{~nm}$ [Fig. 3(a)]. Fig. 3(b) and (c) shows the measured SPIDER signal and the corresponding replica spectra from 380 to $420 \mathrm{~nm}$ before FB (SLM-switch off), respectively, using external reference pulses with energy of $1.2 \mu \mathrm{J} /$ pulse. Intensified reference pulses were obtained from the same amplifier as that for fiber input pulses and were chirped strongly by passing through a highly dispersive glass. The measured spectral envelopes [Fig. 3(b) and (c)] were similar to the fiber output spectrum [Fig. 3(a)], as expected from the principle of the SPIDER [3]. Using these results with a delay time $\tau_{\mathrm{d}}$ of $960 \mathrm{fs}$ and a spectral shear $\Omega / 2 \pi$ of $5.23 \mathrm{THz}$, the spectral phase was reconstructed [see the dashed curve in Fig. 4(a)], and the corresponding negative phase was applied directly and quickly to the SLM by the computer (SLM-switch on). The FB closed loop was repeated twice for better phase compensation. Reconstructed spectral phases and temporal intensity profiles after first and second FBs are shown in Fig. 4(a) and (b), respectively. These results indicate that the asymmetrical phase variation over 25 rad before FB becomes flat after the second FB, and the asymmetrically broadened pulse profile with a duration of over $200 \mathrm{fs}$ before $\mathrm{FB}$ is compressed to $22 \mathrm{fs}$. The compressed pulse is very close to the 20 -fs TL pulse [see the inset of Fig. 4(b)].

2) IPM: We also demonstrated that the SRCSC is significantly useful for compensation of induced phase modulated pulses [15]. An IPM technique based on nonlinear copropagation of two different color ultrashort pulses with a carrier-phase locking in a fiber enables us to broaden the spectral width more efficiently than that in an SPM technique [24]-[29]. However, 


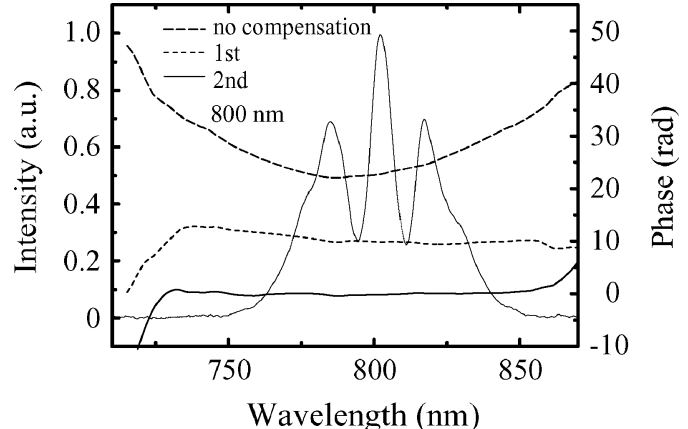

(a)

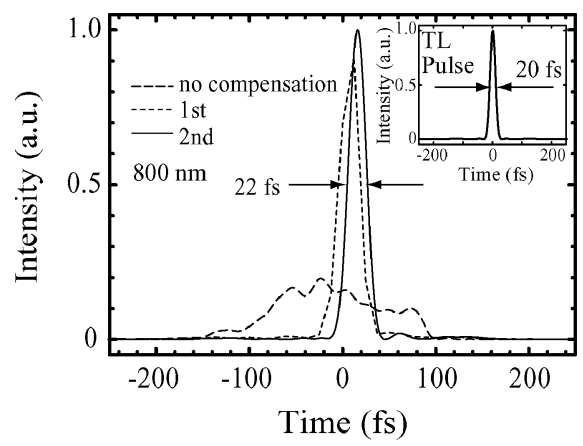

(b)

Fig. 4. SPM experiment. (a) Reconstructed spectral phase before (dashed curve) and after first (dotted curve) and after second FB (solid curve). A thin solid curve is pulse spectrum after second FB. (b) Corresponding reconstructed temporal intensity before (dashed curve) and after first FB (dotted curve) and after second FB (solid curve). Inset shows 19.8-fs Fourier TL pulse.

the spectral phase of the ultrabroadband pulse generated by the dispersive IPM effect is complicated greatly owing to the combined effect of the two-pulse interaction and fiber dispersion. In addition, the peak power is relatively low. Therefore, it is difficult to characterize precisely and compensate for the spectral phase over the whole frequency range.

The experiment for IPM pulse compression was carried out using 800-nm, 80-fs pulses with energy of $64 \mathrm{~nJ} / \mathrm{pulse}$, and $670-\mathrm{nm}, 80-\mathrm{fs}$ pulses with energy of $65 \mathrm{~nJ} / \mathrm{pulse}$, which were generated from a combined system of the regenerative amplifier and the optical parametric amplifier operating at a $1-\mathrm{kHz}$ repletion rate, respectively. The delay time of the $800-\mathrm{nm}$ fundamental pulse with respect to the $640-\mathrm{nm}$ signal pulse was adjusted to overlap in the middle of a fused-silica fiber with a 4-mm length and hence to yield the most efficient IPM effect. The fiber output efficiency was $20 \%$. The output pulse was spectrally broadened from 530 to $880 \mathrm{~nm}$ [Fig. 5(a)] and was directed to the similar SRCSC as that in Fig. 2. Spectral phases and temporal intensity profiles reconstructed from SPIDER signals before FB [Fig. 5(b)] and after the third FB with values of $\tau_{\mathrm{d}}=960 \mathrm{fs}$ and $\Omega / 2 \pi=5.58 \mathrm{THz}$ are shown in Figs. 6 and 7 , respectively. In addition, the group delay as a function of the frequency before FB is shown by a dashed curve in Fig. 6(a). Results indicate that the fiber output pulse has a significantly large and complicated phase variation over $150 \mathrm{rad}$. Correspondingly, the frequency-dependent group delay varing over $500 \mathrm{fs}$ indicates an effective discontinuity ( $\sim 50 \mathrm{fs}$ jump) around $740 \mathrm{~nm}$,

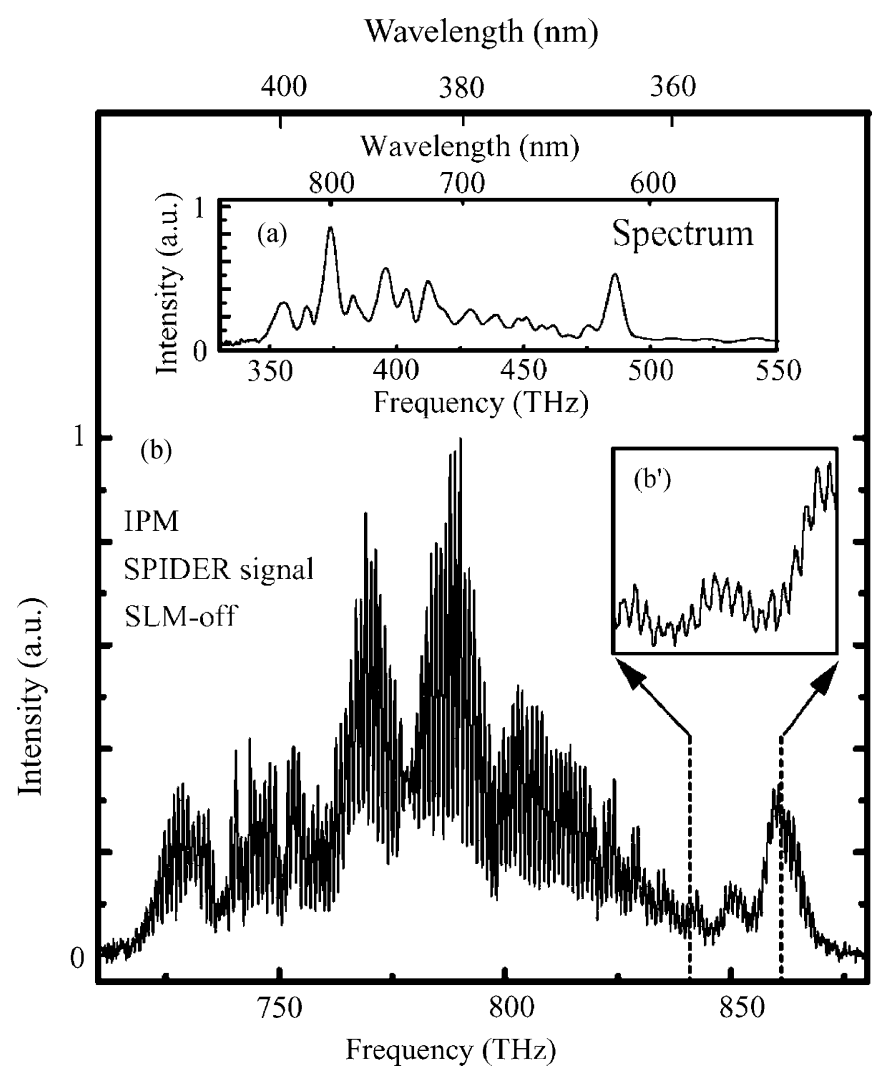

Fig. 5. IPM experiment. (a) Pulse spectrum and (b) [and (b')] SPIDER signal in the SLM-off case.

where the two input pulses spectrally overlap at the fiber output. This is mainly due to the delay time between two pulses at the fiber input and due to their group-velocity mismatch as well as the IPM + SPM effects [15], [27], [30]. In general, such novel behavior of the spectral phase is not compensated for by conventional passive compensators. However, the third FB result (Fig. 7) suggests that even the novel complicated phase behavior is successfully compensated for. That is, the multistructured asymmetric output pulse with a duration of about 500 fs from the fiber was compressed to $7.8 \mathrm{fs}$, which is close to the 4.4-fs TL pulse. The difference is mainly due to imperfect compensations at the low-and high-frequency edges. These occur because the applied phase shift per 1 pixel at the edges exceeds $\pi$ radians. These problems, which resulted in the experiment using the first SRCSC but are not the proper ones in the IPM effect, can be solved using the sophisticated SRCSC where the applied phase is optimized so that the phase modulation load per each pixel is greatly reduced (see Sections III-B1 and Sections IIIC). This experimental result is the first demonstration of optical pulse compression for the white light continuum generated by the IPM effect.

\section{B. Experiments of Photonic Crystal Fiber (PCF) and Tapered Fiber (TF)}

1) $P C F$ : The PCF with unusual dispersion properties enables us to generate efficiently ultrabroadband pulses, using low peak-power pulses produced directly from a laser oscillator 

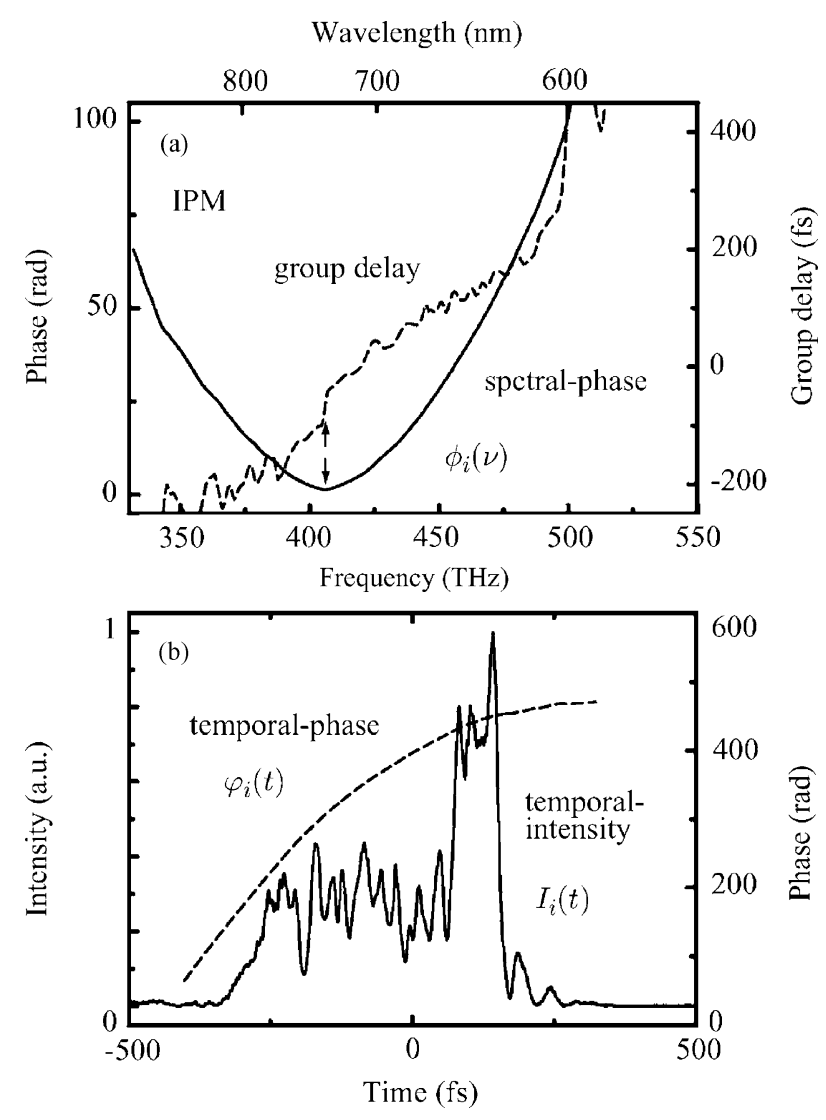

Fig. 6. IPM experiment. (a) Reconstructed spectral phase (solid curve) and spectral group delay (dashed curve) in the SLM-off case. (b) Corresponding reconstructed temporal intensity (solid curve) and temporal phase (dashed curve).

operating at a 75-MHz repetition rate. However, the PCF output pulse exhibits a complicated spectral phase because several nonlinear phenomena occur simultaneously, such as SPM, parametric four-wave mixing, stimulated Raman scattering, high-order soliton formation and collapse, and self-steepening, as well as unusual dispersion profile. Therefore, conventional passive elements such as a combination of a prism pair and chirp mirrors could not compensate for its spectral phase [31]. But, we recently demonstrated that the SRCSC enables us to compensate for such complicated spectral phase and the 12 -fs input pulse was compressed to 6.6 fs [16], as shown below.

The pulse beam from a mode-locked Ti:sapphire laser (12-fs duration, $600-\mathrm{mW}$ average power, $75-\mathrm{MHz}$ repetition rate, and 790-nm center wavelength, with a spectrum from 680 to 920 $\mathrm{nm}$ ) was split with a 1:3 beam splitter. The lower intensity pulse $(150 \mathrm{~mW})$ was passed through a loose-focusing lens for collimation and then directed into the M-SPIDER as an intensified reference chirped pulse. The higher intensity pulse $(450 \mathrm{~mW})$ was coupled into the 3-mm-long PCF [2.6- $\mu \mathrm{m}$ core diameter, 900-nm zero dispersion wavelength (ZDW)]. The effective coupling and transmission efficiency was $20 \%$. The fiber output pulse with a $60-\mathrm{mW}$ average power and a spectral broadening from 620 to $945 \mathrm{~nm}$ [Fig. 8(a)] was directed into the similar SRCSC as shown in Fig. 2 [16]. Fig. 8(b)-(d) shows the
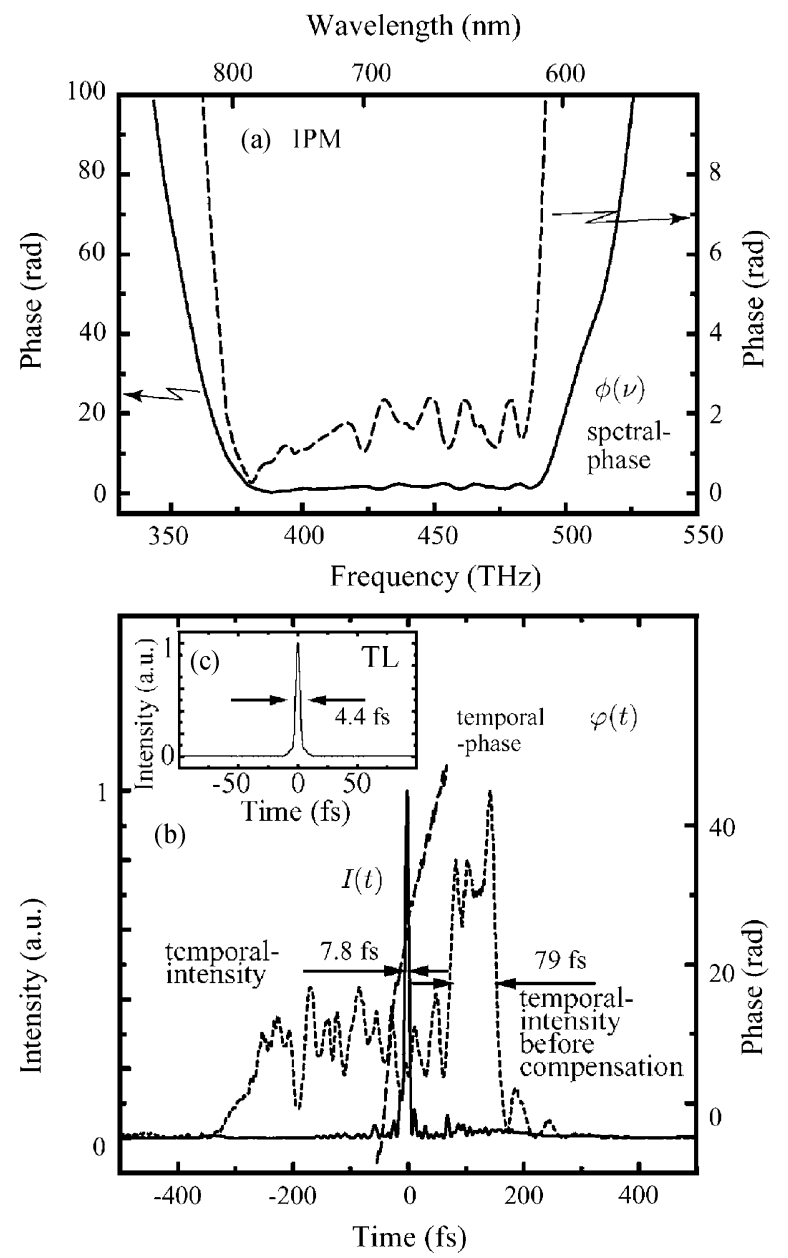

Fig. 7. IPM experiment. (a) Reconstructed spectral phase (solid curve) and its enlarged one (dashed curve) in the SLM-on case. (b) Corresponding reconstructed temporal intensity (solid curve) and temporal phase (dashed curve). A dotted curve shows temporal intensity of fiber output pulse before compensation. (c) Calculated TL pulse profile.

SPIDER signal and the two corresponding replica spectra before compensation (delay time $\tau_{\mathrm{d}}=850 \mathrm{fs}$ and spectral shear $\Omega / 2 \pi=8.24 \mathrm{THz}$ ). Fig. 9(a)-(d) shows reconstructed spectral phases, a pair of the reconstructed temporal intensity and phase profiles before $\mathrm{FB}$, a pair of that after the first $\mathrm{FB}$, and after the second FB, respectively. These results show that the PCF output pulse, which broadens temporally over $150 \mathrm{fs}$ with the complicated spectral phase variation over $30 \mathrm{rad}$, was compressed to $7.1 \mathrm{fs}$ after the first FB and to $6.6 \mathrm{fs}$ after the second FB. The 6.6-fs compressed pulse almost corresponds to the 6.3-fs TL pulse. This is the first complete pulse compression using the PCF in the two-cycle region. The compressed pulse after the second FB was also confirmed by the independent FRAC measurement (Fig. 10).

After the first experiment, the further pulse compression to 5.8 fs (for the 5.0-fs TL pulse) was done using the PCF with a shorter ZDW of $853 \mathrm{~nm}$ and a shorter length of $2 \mathrm{~mm}$ [17].

More recently, a computer simulation study on PCF pulse compression pointed out that degradation of the fiber output coherence between phases from pulse to pulse brings 

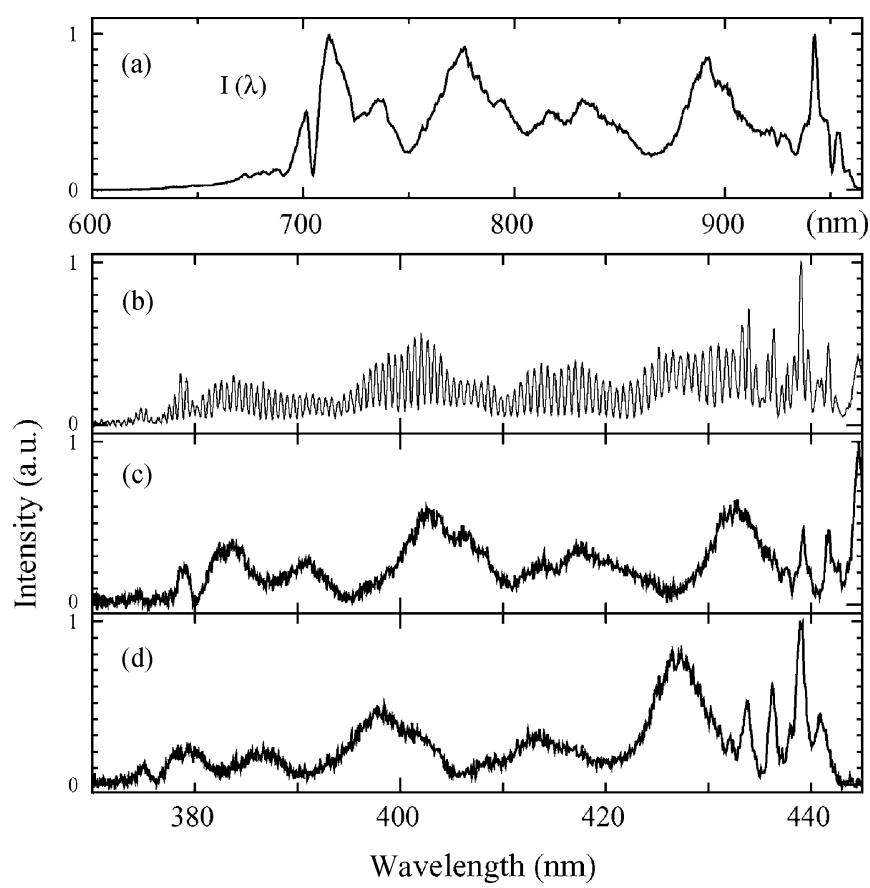

Fig. 8. PCF experiment. SLM-off case (before phase compensation). (a) PCF output spectrum. (b) SPIDER singnal. (c), (d) Replica spectra.

about a severe limit to its compressibility [32]. That is, in the negative-dispersion region, a high-order soliton breaks up to give a fine and complicated spectral structure and a low spectral intensity part. This process is very sensitive to the intensity fluctuation from pulse to pulse of the input and so the spectral phase coherence can easily be degraded. To clarify this problem which becomes more severe for the more efficient compression by the shorter-ZDW PCF, an experiment using the improved SRCSC has been carried out [17]. The main improved point is the reduction of the phase-modulation load per pixel of the SLM by using of a pair of gratings with a larger number of grooves, a shorter blaze wavelength, and a broader bandwidth of silver coating. The 12 -fs pulse with a $480-\mathrm{mW}$ average power was injected into the $1.5-\mathrm{mm}$ long PCF with a 744-nm ZDW, and the $60-\mathrm{mW}$ output pulse was broadened spectrally from 480 to $1000 \mathrm{~nm}$ [curve (i) in Fig. 11(a)]. Measured SPIDER signals before FB and after the first and second FBs $\left(\tau_{\mathrm{d}}=873 \mathrm{fs}\right.$ and $\Omega / 2 \pi=8.20 \mathrm{THz}$ ) are shown in Fig. 11(b)-(d), respectively. However, the fringe visibility in the SPIDER interferograms in the spectral region from 833 to $902 \mathrm{~nm}$ (region B) after the first and second FBs was destroyed. This negative-dispersion region corresponds to the region of the aforementioned soliton breaking up. Therefore, the fine spectral structure and the low-intensity part in this region, where degradation of the phase coherence easily occurs, make it difficult to obtain a good spectral inter-ferogram. This difficulty is greatly enhanced by the deep phase modulation applied by the SLM for phase compensation. This is because the large phase-modulation difference between neighbor pixels results in an artificial sharp modulation of the electric field amplitude spectrum of the fundamental pulse according to the Kramers-Kronig relation. This yields a more complicated structure of the SPIDER signal and hence
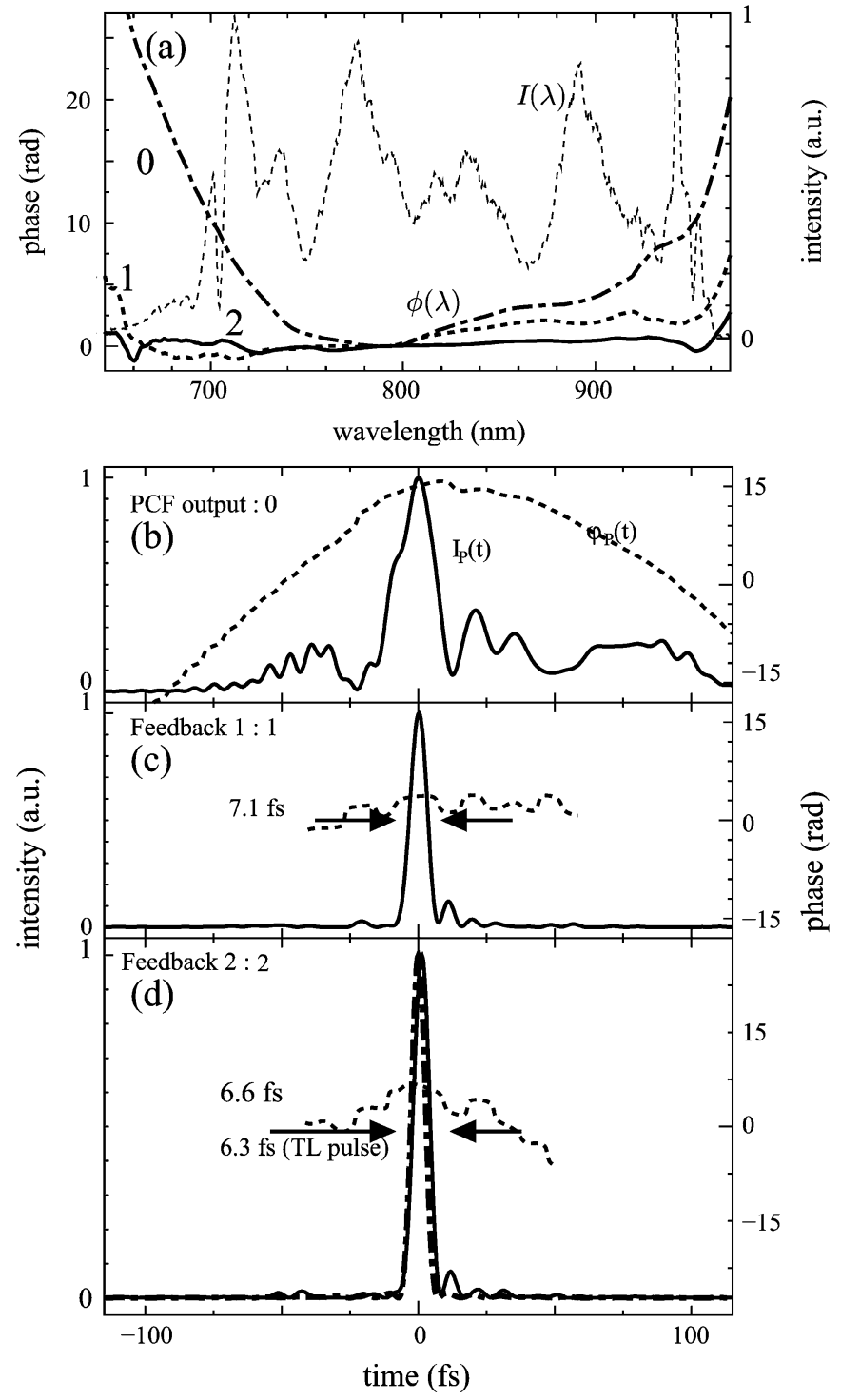

Fig. 9. PCF experiment. (a) Dashed-dotted, dotted, and solid curves are reconstructed spectral phases before compensation and after first FB and after second FB compensations, respectively. A thin dashed curve is SLM-output spectrum after second FB. (b)-(d) Reconstructed temporal intensity profiles (solid curves) and phases (dotted curves) for (b) before compensation and after (c) first FB and (d) second FB compensations. A dashed-dotted curve in (d) is 6.3-fs TL pulse.

the low visibility. That is, visibility in some wavelength region is destroyed. Accordingly, for the undecided part of the spectral phase to be measured in the first and second FB cases we assumed the following: the corresponding spectral phase has the same group-delay dispersion (GDD) as the GDD averaged between the GDD at the lowest frequency edge $\nu_{\mathrm{L}}$ of region $\mathrm{B}$ and that at the highest frequency edge $\nu_{\mathrm{H}}$. In addition, a constant group delay and a constant phase are added at $\nu_{\mathrm{L}}$ so that the spectral phase becomes continuous at $\nu_{\mathrm{L}}$ and $\nu_{\mathrm{H}}$. Spectral phases and temporal intensity profiles obtained for the first and second FBs under such assumption are shown by curves (iii) and (iv) in Figs. 11(a) and 12(b) and (c). The result from Fig. 12(c) indicates that the duration of the main pulse is $4.0 \mathrm{fs}$, while the TL pulse duration is $2.7 \mathrm{fs}$. We also confirmed that a clean $4.9-\mathrm{fs}$ 


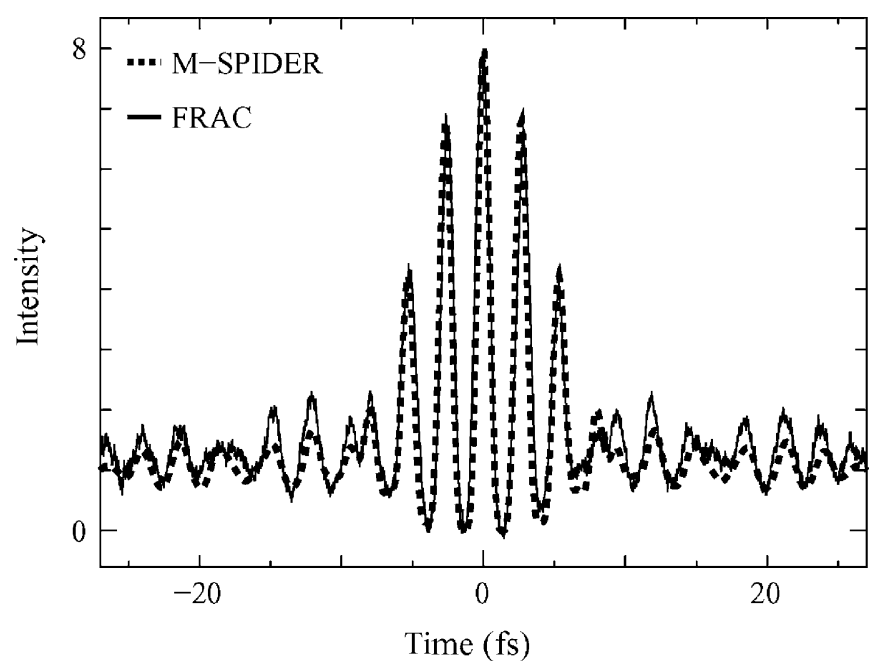

Fig. 10. PCF experiment. FRAC traces of direct measurement (solid curve) and retrieved from M-SPIDER data (dotted curve).

pulse is generated in the spectral region from 480 to $694 \mathrm{~nm}$, where the spectral phase was correctly measured without any assumption [Fig. 12(d)]. The 4.9-fs duration is very close to the corresponding TL pulse duration of 4.7 -fs.

2) $T F$ : Another candidate as the highly efficient pulse compressor is a tapered fused-silica fiber (TF), which has also unusual dispersion properties. We also carried out an experiment of TF pulse compression using the similar SRCSC [14]. The employed TF had a length of $74 \mathrm{~mm}$, a waist length of $32.5 \mathrm{~mm}$, a waist diameter of $2 \mu \mathrm{m}, 9-\mu \mathrm{m}$ long core diameters at input and output sides, and an evaluated ZDW of $710 \mathrm{~nm}$. The 185 -fs pulse beam, which has an average power of $100 \mathrm{~mW}$ and a center wavelength of $785 \mathrm{~nm}$ at a $100-\mathrm{MHz}$ repetition rate, was compressed to 16 fs after the first FB compensation. Furthermore, more recently, shorter pulse compression to $8.4 \mathrm{fs}$ was demonstrated for 12-fs input pulses with an average power of $200 \mathrm{~mW}$ at a repetition rate of $75 \mathrm{MHz}$ [33].

\section{Gas-Filled Hollow Fiber Experiment: Quasi-Monocycle Pulse Compression}

High pressure noble gas filled hollow fiber (HF) is an attractive way of producing an ultrabroadband spectrum (over one octave) with intense laser pulses. We demonstrated that the improved SRCSC enables us to compress to sub-3 fs in the monocycle region [7], [8].

The experimental setup is shown in Fig. 13. The 30-fs, $135-\mu \mathrm{J}$ pulse from an amplifier operating at a $1-\mathrm{kHz}$ repetition rate (a center wavelength of $790 \mathrm{~nm}$ ) was propagated in a $34-\mathrm{cm}$ long hollow fiber (a core diameter of $100 \mu \mathrm{m}$ ) filled with 3.0-atm argon gas. The ultrabroadband output pulse [curve (iv) in Fig. 14(b): 460-1060 nm corresponding to a 369-THz bandwidth, a center wavelength of $580 \mathrm{~nm}$ ] produced by the SPM was guided into the SRCSC. The output pulse energy from the hollow fiber and that from the phase manipulator were $18 \mu \mathrm{J}$ and $0.5 \mu \mathrm{J}$, respectively. The reason why the throughput is small is the use of several reflections of Al-coated mirrors for output beam guides. The throughput can then be improved. The pulse
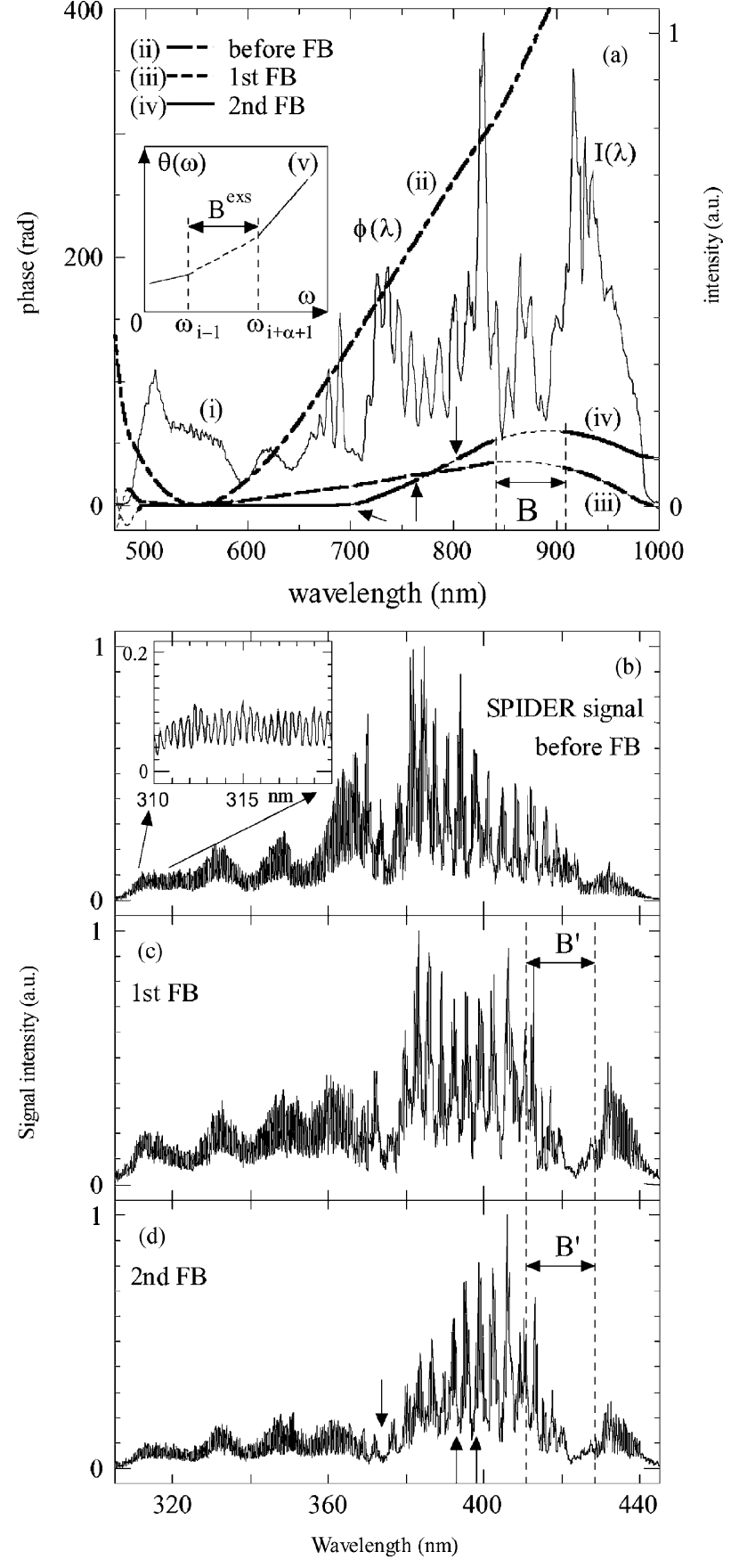

Fig. 11. PCF experiment. (a) Dashed-dotted (ii), dashed (iii), and solid (iv) curves are reconstructed spectral phases before compensation, after first and second FB compensations for a PCF (ZDW $744 \mathrm{~nm}$, length $1.5 \mathrm{~mm}$, core diameter $1.8 \mu \mathrm{m}$ ), respectively. The dotted-curve parts in (iii) and (iv) are estimated spectral phases. The thin curve (i) is the SLM-output spectrum after second FB. (v) Schematic drawing of spectral phase difference $\theta(\omega)$ for undecided part. SPIDER signals for (b) before FB compensation and after (c) first and (d) second FB compensations.

with an energy of $3.7-\mu \mathrm{J}$, which was obtained by splitting the pulse from the same amplifier, was employed as an external intensified reference pulse for the M-SPIDER.

To compensate for the spectral phase perfectly and to get a clean TL pulse in the monocycle region, we directed our attention to the phase manipulator with the SLM regarding the 


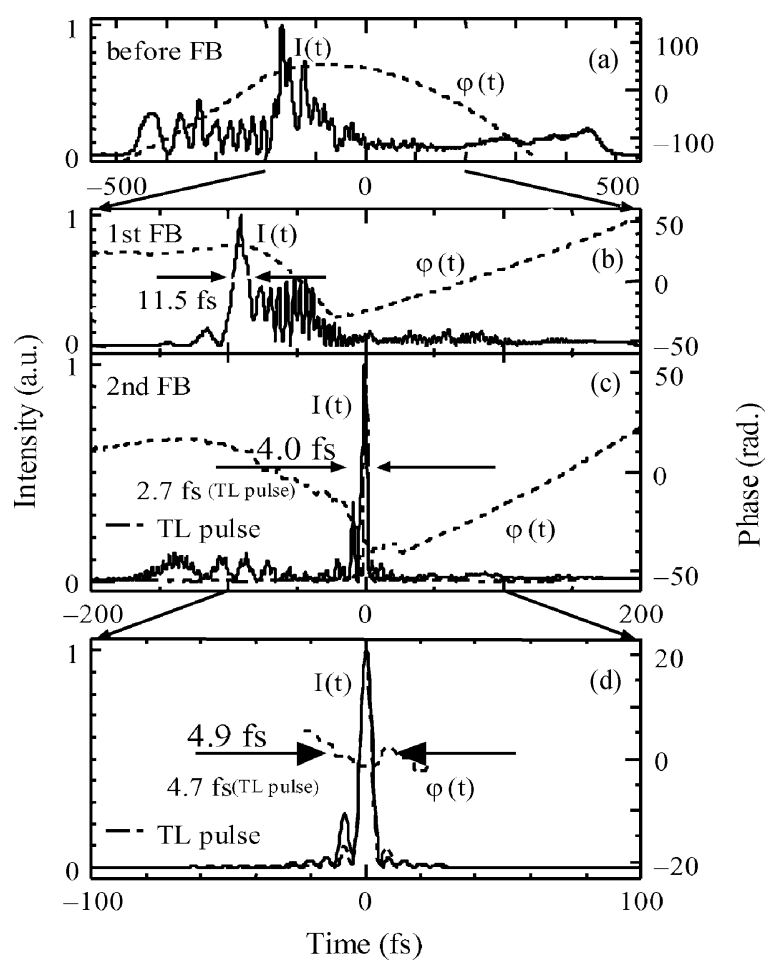

Fig. 12. PCF experiment. Reconstructed temporal intensity profiles (solid curves) and phase (dotted curves) for (a) compensation and after (b) first and (c) second FB compensations in the experiment of PCF with ZDW of $744 \mathrm{~nm}$. The dashed-dotted curve in (c) is the 2.7-fs TL pulse. (d) Reconstructed temporal intensity profiles (solid curve) and phase (dotted curve) in spectral region from 480 to $694 \mathrm{~nm}$ after second FB. The dashed-dotted curve is the 4.7-fs TL pulse.

Feedback system

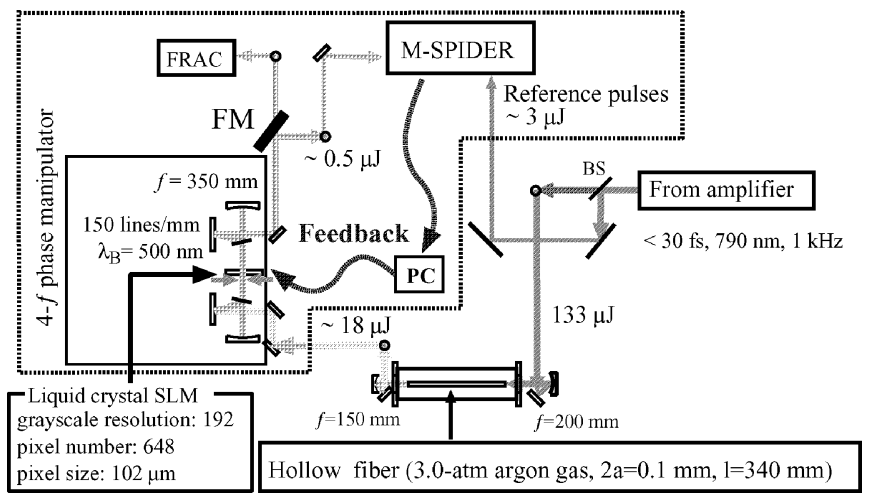

Fig. 13. HF experiment. Experimental setup for the generation of 2.8-fs 1.5cycle TL pulses.

following [8]. First, the initial alignment of the $4-f$ configuration was performed in accuracy of $|\Delta z|<0.1 \mathrm{~mm}$, where $\Delta z$ is the difference between the twice of the focusing length $f$ of CM1 or CM2 and the sum of the distance between $\mathrm{G} 1$ and CM1 and that between $\mathrm{CM} 2$ and $\mathrm{G} 2$, to avoid spatial dispersion for the over-one-octave spectrum. This was done by observing the pattern of the interference between second-order and third-order diffracted beams from G2 [8]. Second, the phase modulation load per pixel of the SLM was greatly reduced by selecting a suitable combination of a constant phase $\phi_{\mathrm{C} 0}$ and a group delay $\dot{\phi}_{\mathrm{C}}\left(\omega_{0}\right)$ in the spectral phase $\phi_{\mathrm{C}}(\omega)$, which was applied to the
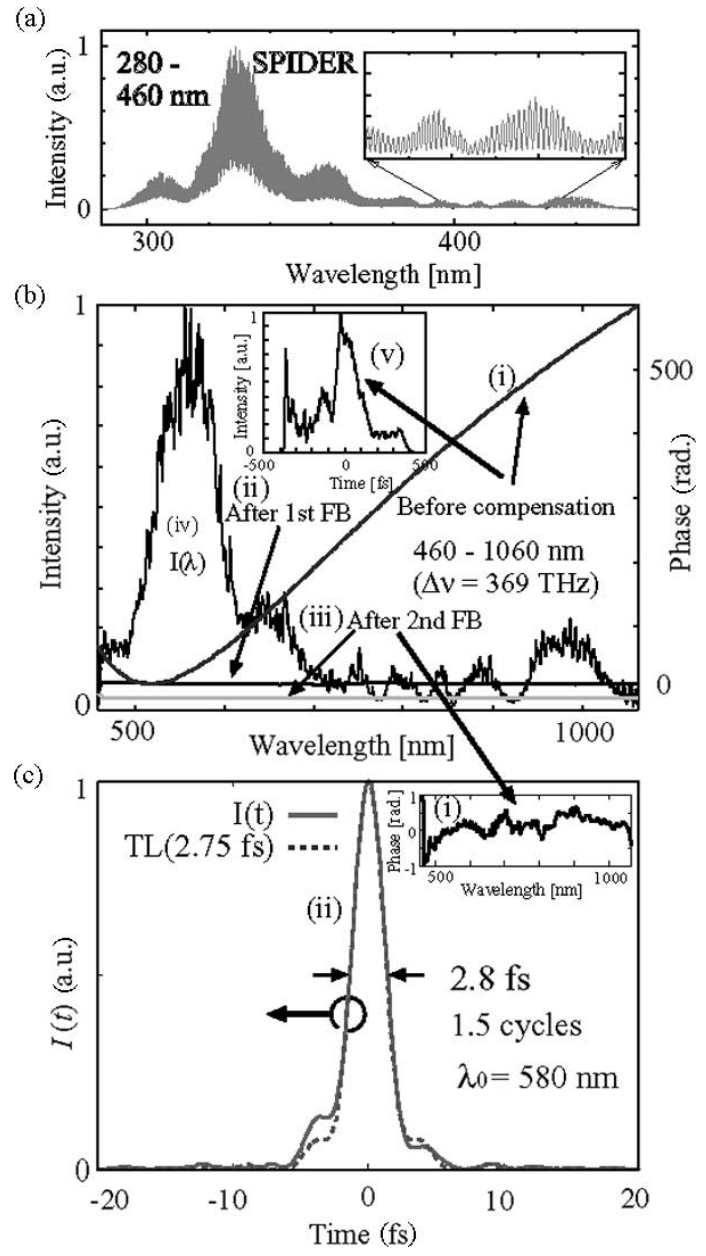

Fig. 14. HF experiment. Generated 2.8-fs TL pulses with a single, clean 1.5cycle waveform. (a) Observed M-SPIDER signal over the spectral range from 280 to $480 \mathrm{~nm}$ with a good signal-to-noise ratio before phase compensation. (b) Reconstructed spectral phase (i) and temporal intensity profile (v) before phase compensation, reconstructed spectral phases after the first [(ii) after first FB] and second [(iii) after second FB] FB phase compensations, and intensity spectrum $I(\lambda)$ (iv) with a spectral width from 460 to $1060 \mathrm{~nm}$ (a center wavelength of $\lambda_{0}=580 \mathrm{~nm}$ ) corresponding to a bandwidth of $\Delta \nu=369 \mathrm{THz}$. The spectral phase after second FB is flat over the whole spectral region. (c) After the second $\mathrm{FB}$, reconstructed temporal intensity profile $I(t)$ (ii) with an FWHM of $2.8 \mathrm{fs}$ and its corresponding spectral phase (i) which is the enlarged version of curve (iii) in (b). A dotted line is a Fourier-TL ideal pulse with an FWHM of $2.75 \mathrm{fs}$, which is calculated from the intensity spectrum of curve (iv) in (b). Generated 1.5 -cycle pulses indicating a single, clean waveform are extremely close to TL pulses. This means that perfect compensation over the one-octave bandwidth was performed successfully.

SLM for compensation [8]. These efforts allow us to manipulate the spectral phase over the more-than one octave very accurately.

Fig. 14(a) shows the SPIDER signal with a high signal-tonoise ratio in the whole spectral range from 280 to $460 \mathrm{~nm}$, which was measured before phase compensation. The spectral shear $\Omega / 2 \pi$ and the delay time $\tau_{\mathrm{d}}$ were $4.24 \mathrm{THz}$ and $930 \mathrm{fs}$, respectively. Fig. 14(b) shows reconstructed spectral phases before compensation (over 500-rad variation) and after first and second FB compensations. In addition, the reconstructed temporal intensity profile before compensation is shown to be complicated and broadened from -500 to +500 fs [curve (v) in Fig. 14(b)]. Furthermore, the spectral phase after the second FB 


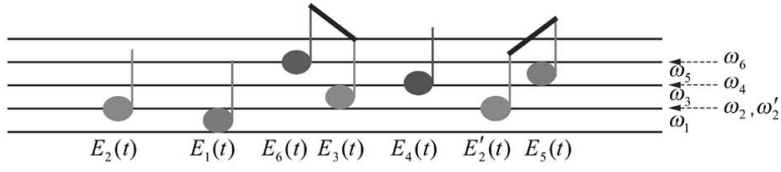

$\mathbf{E}(\mathbf{r}, t)=\frac{1}{2} \mathbf{e}_{1}(t, \mathbf{r}) A_{1}(t, \mathbf{r}) \exp \left\{i\left[\omega_{1} t-\mathbf{k}_{1}(t, \mathbf{r}) \cdot \mathbf{r}+\varphi_{1}(t, \mathbf{r})\right]\right\}+\cdots \quad \cdots+c . c$.

Fig. 15. Intuitive concept of optical-wave musical instrument and/or optical function generator, that is, arbitrary time-sequential, automatic manipulator of optical electric field wavepackets.

is almost completely flattened [curve (iii) in Fig. 14(b); curve (i) in Fig. 14(c) is its enlarged version]. The corresponding temporal intensity profile [curve (ii) in Fig. 14(c)] indicates that subpulses are completely eliminated and the duration (a full-width at half-maximum) is $2.8 \mathrm{fs}$. This corresponds to 1.5 optical cycles of the carrier wave with a 580-nm center wavelength, and the single clean profile is almost the same as its ideal TL pulse with a 2.75-fs duration [dotted curve in Fig. 14(c)]. To the best of our knowledge, this is the shortest TL pulse with a single clean profile, which was achieved in the visible to near-infrared region.

Recently, we applied the locking technique of the carrierenvelope phase (CEP) to the aforementioned optical system generating quasi-monocycle pulses [34]. The result of the preliminary experiment using our home-made locking device has showed that the CEP of 3.3-fs, 1.7-cycle optical pulses at a 1-kHz repetition rate is significantly stabilized within $\pi / 2 \operatorname{rad}$ for $20 \mathrm{~s}$.

\section{OUTLOOK}

Our extensive results suggest that the developed sophisticated spectral-phase feedback technique making the method of the cumbersome Taylor expansion unnecessary can be applied for nonlinear chirp compensation of any unknown or fluctuated spectral phase even exceeding a one octave bandwidth in the quasi-real-time mode. Furthermore, the self-recognition type of the technique has potentialities of a useful tool in generating monocycle pulses and in engineering optical electric field wave packets time sequentially (such as acoustic waves by a musical instrument; optical-wave musical instrument: [35, Fig. 15], even for those who are not familiar with ultrafast optics. We believe that the SRCSC will become a trigger to bring about a new field where spatiotemporal manipulation and synthesis of the phase, amplitude, frequency, polarization, and beam direction of temporal electric field wave packet play an essential role. For example, the control of the electric-field wave packet but not the pulse intensity envelope is significantly important for the production of single attosecond pulses by the high-order harmonic generation using CEP controlled high-intensity pulses [36].

\section{REFERENCES}

[1] K. Yamane, T. Kito, R. Morita, and M. Yamashita, "Experimental and theoretical demonstration of validity and limitations in fringe-resolved autocorrelation measurements for pulses of few optical cycles," Opt. Express, vol. 12, pp. 2762-2733, 2004.

[2] Z. Cheng, A. Fürbach, S. Sartania, M. Lenzner, C. Spielmann, and F. Krausz, "Amplitude and chirp charactrization of high-power laser pulses in the 5-fs regime," Opt. Lett., vol. 24, pp. 247-249, 1999.
[3] C. Iaconis and I. A. Walmsley, "Self-referencing spectral interferometry for measuring ultrashort optical pulses," IEEE J. Quantum Electron., vol. 35, no. 4, pp. 501-509, Apr. 1999.

[4] B. Schenkel, J. Biegert, U. Keller, C. Vozzi, M. Nisoli, G. Sansone, S. Stagira, S. De Silvestri, and O. Svelto, "Generation of 3.8-fs pulses from adaptive compression of a cascaded hollow fiber seupercontinuum," Opt. Lett., vol. 28, pp. 1987-1989, 2003.

[5] C. P. Hauri, W. Kornelis, F. W. Helbing, A. Couairon, A. Mysyrowicz, J. Biegert, and U. Keller, "Genration of intense, carrier-envelope phaselocked laser pulses through filamentation," Appl. Phys. B, vol. 79, pp. 673677, 2004.

[6] M. Hirasawa, N. Nakagawa, K. Yamamoto, R. Morita, H. Shigekawa, and M. Yamashita, "Sensitivity improvement of spectral phase interferometry for direct electric-field reconstruction for the characterization of lowintensity femtosecond pulses," Appl. Phys. B, vol. 74, pp. S225-S229, 2002.

[7] K. Yamane, Z. Zhang, K. Oka, R. Morita, M. Yamashita, and A. Suguro, "Optical pulse compression to $3.4 \mathrm{fs}$ in the monocycle region by feedback phase compensation," Opt. Lett., vol. 28, pp. 2258-2260, 2003.

[8] K. Yamane, T. Kito, R. Morita, and M. Yamashita, "2.8-fs transformlimited optical-pulse generation in the monocycle region," in Proc. CLEO 2004, vol. 2, pp. 1045-1047.

[9] A. M. Weiner, D. F. Leaird, J. S. Patel, and J. R. Wullert, "Programmable femtosecond pulses shaping by use of a multielement liquid-crystal phase modulator," Opt. Lett., vol. 15, pp. 326-328, 1990.

[10] F. Verluise, V. Loude, Z. Cheng, Ch. Spielmann, and P. Tournois, "Amplitude and phase control of ultrashort pulses by use of on acousto-optic programmable despersive filter: Pulse compression and shaping," Opt. Lett., vol. 25, pp. 575-577, 2000.

[11] A. Baltuška, T. Fuji, and T. Kobayashi, "Visible pulse compression to $4 \mathrm{fs}$ by optical parametric amplification and programmble dispersion control," Opt. Lett., vol. 27, pp. 306-308, 2002.

[12] D. Yelin, D. Meshulach, and Y. Silberberg, "Adaptive femtosecond pulse compression," Opt. Lett., vol. 22, pp. 1793-1795, 1997.

[13] M. Hirasawa, N. Nakagawa, K. Yamamoto, A. Hashimoto, R. Morita, A. Suguro, H. Shigekawa, and M. Yamashita, in Tech. Dig. Conf. Lasers and Electro-Optics 2002 (CLEO 2002), Washington, DC, 2002, p. 356.

[14] M. Adachi, M. Hirasawa, A. Suguro, N. Karasawa, S. Kobayashi, R. Morita, and M. Yamashita, "Spectral-phase characterization and adapted compensation of strongly chirped pulses from a tapered fiber," Jpn. J. Appl. Phys., vol. 42, pp. L24-L26, 2003.

[15] M. Yamashita, M. Hirasawa, N. Nakagawa, K. Yamamoto, K. Oka, R. Morita, and A. Suguro, "Pulse compression of white-light continuum generated by induced phase modulation in a conventional glass fiber," $J$. Opt. Soc. Amer. B, Opt. Phys., vol. 21, pp. 458-462, 2004.

[16] M. Adachi, K. Yamane, R. Morita, and M. Yamashita, "Pulse compression using direct feedback of the spectral phase from photonic crystal fiber output without the need for the Taylor expansion method," IEEE Photonics Technol. Lett., vol. 16, no. 8, pp. 1951-1953, Aug. 2004.

[17] — "Sub-5-fs pulse compression of laser output using photonic crystal fiber with short zero-dispersion wavelength," Jpn. J. Appl. Phys., vol. 44, pp. L1423-L1425, 2005.

[18] E. Goulielmakis, M. Ulberacker, R. Kienberger, A. Baltuška, V. Yakovlev, A. Scrinzi, T. Westerwalbestoh, U. Kleinberg, U. Heinzmann, M. Dresher, and F. Krausz, "Direct measurement of light waves," Science, vol. 305, pp. 1267-1269, 2004.

[19] L. Xu, N. Nakagawa, R. Morita, H. Shigekawa, and M. Yamashita, "Programmable chirp compensation for 6-fs pulse generation with a prismpair-formed pulse shaper," IEEE J. Quantum Electron., vol. 36, no. 8, pp. 893-899, Aug. 2000.

[20] L. Xu, L. Li, N. Nakagawa, R. Morita, and M. Yamashita, "Application of a spatial light modulator for programmable optical pulse compression to the sub-6-fs regime," IEEE Photon. Technol. Lett., vol. 12, no. 11, pp. 1540-1542, Nov. 2000.

[21] N. Karasawa, L. Li, A. Suguro, H. Shigekawa, R. Morita, and M. Yamashita, "Optical pulse compression to 5.0 fs by use of only a spatial light modulator for phase compensation," J. Opt. Soc. Amer. B, Opt. Phys., vol. 18, pp. 1742-1746, 2001.

[22] Y. Deng, Z. Wu, L. Chai, C. Wang, K. Yamane, R. Morita, M. Yamashita, and Z. Zhang, "Wavelet-transform analysis of spectral shearing interferometry for phase reconstruction of femtosecond optical pulses," Opt Express, vol. 13, pp. 2120-2126, 2005. 
[23] K. Narita, M. Adachi, R. Morita, and M. Yamashita, "Complete automatic phase compensation for the generation of a-few-cycle pulses," in Ultrafast Optics V, S. Watanabe, Ed. Berlin, Germany: Springer-Verlag, 2005.

[24] M. Yamashita, H. Sone, and R. Morita, "Proposal for generation of a coherent pulse ultra-broadened from near-infrared to near-ultraviolet and its monocyclization," Jpn. J. Appl. Phys., vol. 35, pp. L1194-L1197, 1996.

[25] M. Yamashita, H. Sone, R. Morita, and H. Shigekawa, "Generation of monocycle-like optical pulses using induced-phase modulation between two-color femtosecond pulses with carrier phase locking," IEEE J. Quantum Electron., vol. 34, no. 11, pp. 2145-2149, Nov. 1998.

[26] N. Karasawa, R. Morita, L. Xu, H. Shigekawa, and M. Yamashita, "Theory of ultrabroadband optical pulse generation by induced-phase modulation in a gas-filled hollow waveguide," J. Opt. Soc. Amer. B, Opt.Phys., vol. 16, pp. 662-668, 1999.

[27] L. Xu, N. Karasawa, N. Nakagawa, R. Morita, H. Shigekawa, and M. Yamashita, "Experimental generation of an ultra-broad spectrum based on induced-phase modulation in a single-mode glass fiber," Opt. Commun., vol. 162, pp. 256-260, 1999.

[28] N. Karasawa, S. Nakamura, N. Nakagawa, M. Shibata, R. Morita, H. Shigekawa, and M. Yamashita, "Comparison between theory and experiment of nonlinear propagation for a-few-cycle and ultrabroadband optical pulses in a fused silica fiber," IEEE J. Quantum Electron., vol. 37, no. 3, pp. 398-404, Mar. 2001.

[29] N. Karasawa, R. Morita, H. Shigekawa, and M. Yamashita, "Generation of intense ultrabroadband optical pulses by induced phase modulation in an argon-filled single-mode hollow waveguide," Opt. Lett., vol. 25, pp. 183-185, 2000.

[30] M. Shibata, M. Hirasawa, N. Nakagawa, R. Morita, A. Suguro, H. Shigekawa, and M. Yamashita, "Experimental demonstration of phasedispersion compensation for ultrabroadband femtosecond optical pulses generated by induced-phase modulation," Appl. Phys. B, vol. 74, pp. S291S294, 2002.

[31] S. Lakó, J. Seres, P. Apai, J. Balázs, R. S. Windler, and R. Szipöcs, "Pulse compression of nanojoule pulses in the visible using microstructure optical fiber and dispersion compensation," Appl. Phys. B, vol. 76, pp. 267-275, 2003.

[32] J. M. Dudley and S. Coen, "Fundamental limits to few-cycle pulse generation from compression of supercontinuum spectra generated in photonic crystal fiber," Opt. Express, vol. 12, pp. 2423-2428, 2004.

[33] M. Adachi, K. Yamane, R. Morita, and M. Yamashita, in Ultrafast Phenomena XIV, T. Kobayashi et al., Ed. Berlin, Germany: Springer-Verlag 2004, pp. 49-51.

[34] K. Yamane, T. Naoi, R. Morita, K. Sugiyama, M. Kitano, and M. Yamashita, "Carrier-envelope-phase stabilized 3.3-fs optical pulses in the monocycle region," in Tech. Dig. CLEO 2005, Washington, DC, Paper JThE 18.

[35] M. Yamashita, H. Shigekawa, and R. Morita, Monocycle Photonics and Optical Scanning Tunneling Microscopy. Berlin, Germany: SpringerVerlag, 2005.

[36] A. Baltuška, T. Udem, M. Ulberaker, M. Hentschel, E. Goullelmakls, C. Gohle, R. Holzwarth, V. S. Yukovlev, A. Schrinzl, T. W. Hänsch, and F. Krausz, "Attosecond control of electronic processes by intense light fields," Nature, vol. 421, pp. 611-615, 2003.

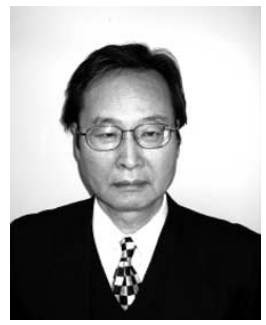

Mikio Yamashita was born in Kyoto, Japan, on February 15, 1946. He received the M.S. and Ph.D. degrees from the Kyoto University, Kyoto, Japan, in 1970 and 1976, respectively, both in electronics engineering.

In 1978, he was a Visiting Research Associate at Imperial College, London, U.K. From 1970 to 1991, he was with the Electrotechnical Laboratory, Tokyo and Tsukuba, Japan. Since 1991, he has been with the Department of Applied Physics, Hokkaido University, Sapporo, Japan, as a Professor, and is currently the Head of the Department. His current research interests are in monocycle photonics, attosecond optics, and their applications including biophotonics.

Dr. Yamashita is a member of the Japan Society of Applied Physics, Physical Society of Japan, Laser Society of Japan, Biophysical Society of Japan, and Optical Society of America.

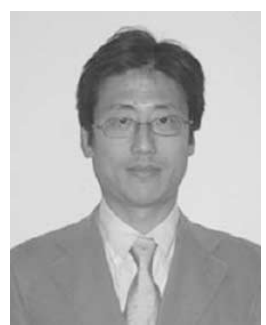

Keisaku Yamane was born in Kyoto, Japan, in 1970. $\mathrm{He}$ received the $\mathrm{Ph}$.D. degree in electronics engineering from Kyoto University, Kyoto, Japan, in 2005.

In 2005, he joined Kawasaki Semiconductor Technology World Corporation, Fukui, Japan, and currently works on diode-pumped solid-state lasers (DPSSL).

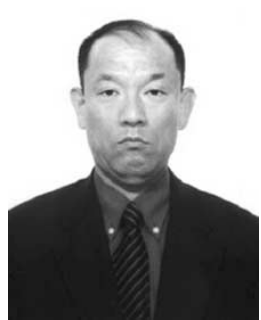

Ryuji Morita was born in Mie, Japan, in 1962. He received the B.E. degree in applied mathematics and information physics, in 1985, and the M.E. and Ph.D. degrees in applied physics, in 1987 and 1990, respectively, from The University of Tokyo, Tokyo, Japan.

From 1990 to 1992, he was a Visiting Scientist at the Laboratoire de Bagnuex, Centre National d'Etudes des Telecommunications (CNET), Bagneux, France. In 1992, he joined Hokkaido University, Sapporo, Japan, where he is currently a Professor in the Department of Applied Physics. His research interests include monocycle optical pulse generation and characterization and application of coherent ultrabroadband optical pulses.

Dr. Morita is a member of the Japan Society of Applied Physics and of the Optical Society of America. 